\title{
A meta-analysis of nivolumab for the treatment of advanced non-small-cell lung cancer
}

This article was published in the following Dove Press journal:

OncoTargets and Therapy

\author{
Shuo Chen \\ Bin $\mathrm{Hu}$ \\ Hui Li \\ Department of Thoracic Surgery, \\ Beijing Chao-Yang Hospital, Capital \\ Medical University, Beijing 100020, \\ People's Republic of China
}

\begin{abstract}
Background: Non-small-cell lung cancer (NSCLC) is often associated with rapid progression following standard chemotherapy. Nivolumab, an inhibitor of PD-1/PD-L1, is reported to have potential efficacy for the treatment NSCLC.

Objective: The purpose of this meta-analysis was to systematically evaluate the efficacy and safety of nivolumab in patients with advanced NSCLC.

Methods: Online electronic databases were searched in June 2017, including: PubMed, Embase, and the Cochrane Library. Randomized controlled trials were included that compared nivolumab to chemotherapy in NSCLC patients with regard to oncological outcome profiles. Review Manager Version 5.3 software was used.

Results: Three studies were included in this analysis, comprising 1,395 patients with NSCLC, of whom 698 received nivolumab and 697 received chemotherapy without nivolumab. The pooled hazard ratios for overall survival (OS) and prolonged progression-free survival (PFS) were 0.77 (95\% CI: $0.57-1.03 ; P=0.08)$ and 0.88 (95\% CI: 0.64-1.20; $P=0.41)$, respectively. The pooled odds ratio for overall response rate was 1.40 (95\% CI: $0.66-2.96 ; P=0.39)$, indicating that no benefit with nivolumab was found for OS, PFS, or overall response rate. However, the odds ratio for treatment-related adverse events, grades 3 or 4 , between the patients who received nivolumab and chemotherapy was 0.13 (95\% CI: $0.09-0.17 ; P<0.00001)$. For patients with a PD-L1 expression level of 5\% or more, no difference was observed in PFS (95\% CI: 0.70-1.00; $P=0.05$ ) and OS benefit (95\% CI: $0.34-1.15 ; P=0.13$ ) between the groups.

Conclusion: These data demonstrate no clinical survival benefit with nivolumab for NSCLC patients, even in a subpopulation of patients with levels of PD-L1>5\%. However, nivolumab had a more favorable safety profile than chemotherapy. Future investigations are needed to determine whether the efficacy of nivolumab can be improved.
\end{abstract}

Keywords: non-small-cell lung cancer, PD-1, nivolumab, meta-analysis

\section{Introduction}

Lung cancer continues to be a major health burden, with most cases diagnosed as non-small-cell lung cancer (NSCLC). This category includes nonsquamous (70\%) and squamous (30\%) histological subtypes. ${ }^{1-3}$ Detection of early-stage NSCLC is often associated with a good prognosis.

However, the majority of patients have advanced, recurrent, or metastatic disease. ${ }^{4}$ Prognosis for these patients is very poor. Although platinum-based doublet treatments are still approved as first-line treatment options for NSCLC, ${ }^{5}$ cancer-associated, immune-targeted therapies have been shown to have survival benefit. ${ }^{6,7}$

The PD-1 pathway inhibits immune responses, and it plays a critical role in many cancer types, including NSCLC. Different from chemotherapy, blockade of the PD-1 
pathway can result in durable antitumor activity, significantly increasing long-term survival..$^{8-12}$

Nivolumab (Opdivo ${ }^{\circledR}$; Bristol-Myers Squibb, Princeton, NJ, USA), a PD-1 immune checkpoint inhibitor, was the first immune checkpoint inhibitor approved for metastatic NSCLC, for use after the failure of platinum-based chemotherapy. ${ }^{13}$ The first trial was the CheckMate-017 trial, ${ }^{14}$ in which nivolumab was shown to improve overall survival (OS), progression-free survival (PFS), and the overall response rate (ORR) over docetaxel. For the second trial, CheckMate-057 trial, ${ }^{15}$ OS with nivolumab was less effective than docetaxel. For the CheckMate-026 trial, ${ }^{16}$ survival efficacy with nivolumab was less than the investigator's choice chemotherapy.

In that effective management of NSCLC with nivolumab has been controversial and with conflicting results, the objective of this meta-analysis was to evaluate the oncological outcomes of nivolumab therapy in pretreated advanced NSCLC.

\section{Materials and methods}

\section{Search strategy}

PubMed, Embase, and Cochrane Library databases were searched up to June 2017 by two independent investigators with the following keywords: "non-small cell lung cancer," and "Programmed cell death-1," and "nivolumab." No limitation was used during the literature search. The references of eligible studies were hand-searched for additional studies.

Ethics Committee approval was waived because this study did not involve any human participants or animals.

\section{Eligibility criteria}

Eligible studies for this meta-analysis met the following criteria: 1) the study was designed as a randomized control trial (RCT); 2) the study enrolled NSCLC patients treated with nivolumab and as well the investigator's choice chemotherapy; 3) outcomes of interest were efficacy (survival, tumor response), drug toxicity (incidence of severe adverse effects [SAEs]), and hazard ratios with 95\% CIs were provided. Studies with the most complete outcomes data were included.

\section{Quality assessment}

The quality of the retrieved studies was rated independently by two of the authors. The risk of bias items recommended by The Cochrane Handbookfor Systematic Reviews of Interventions were chosen.

\section{Data extraction}

Data were extracted by two authors independently. Disagreements were resolved by consensus. For each of the eligible studies, the main categories were based on the following: name of the first author, year of publication, study type, trial name, stage, histology, PD-L1 tumor expression level, treatment regimen, and endpoints of interest. Corresponding variables were adjusted and risk estimates of mortality with 95\% CIs were assessed.

\section{Statistical analysis}

The safety of anti-PD-1/PD-L1 was based on data from RCTs. The endpoints of interest for the pooled analyses were OS, PFS, ORR, and SAE data.

Sensitivity analysis, based on the heterogeneity betweenstudies, was examined using the $I^{2}$ statistic. ${ }^{17}$ Studies with an $I^{2} \geq 50 \%$ were considered to have moderate to high heterogeneity, $I^{2}<50 \%$ were considered to have low heterogeneity. ${ }^{18}$ Summary hazard ratios were calculated by using fixed-effect models when there was low heterogeneity among the studies. Otherwise, random-effect models were used. A $P$-value $<0.05$ was considered statistically significant. Statistical analyses were conducted using Review Manager Version 5.3 software (Revman; The Cochrane Collaboration, Oxford, UK). Meta-analyses are shown as forest plots. The Begg test and the Egger test were used to assess publication bias.

\section{Results \\ Overview of literature search and study characteristics}

By literature search, a total of 351 studies were identified. Of these, 13 studies were evaluated by reading the full article. Some of these studies did not report sufficiently detailed data and only three RCTs ${ }^{14-16}$ met the criteria for inclusion. The search process is described in Figure 1.

All included studies were based on moderate- to highquality evidence. Table 1 provides a brief description of the eligible studies, with some detail.

\section{Clinical and methodological heterogeneity}

Pooled analysis of PFS comparing the addition of nivolumab with chemotherapy

Pooling the PFS data from all three studies ${ }^{14-16}$ showed that nivolumab did not lead to PFS benefit (odds ratio [OR]: 0.88 , 95\% CI: $0.64-1.20, P=0.41)$ compared with chemotherapy (Figure 2).

\section{Pooled analysis of OS comparing the addition of} nivolumab with chemotherapy

A random-effects model was used to pool the OS data, ${ }^{14-16}$ since heterogeneity across the studies was significantly high. 


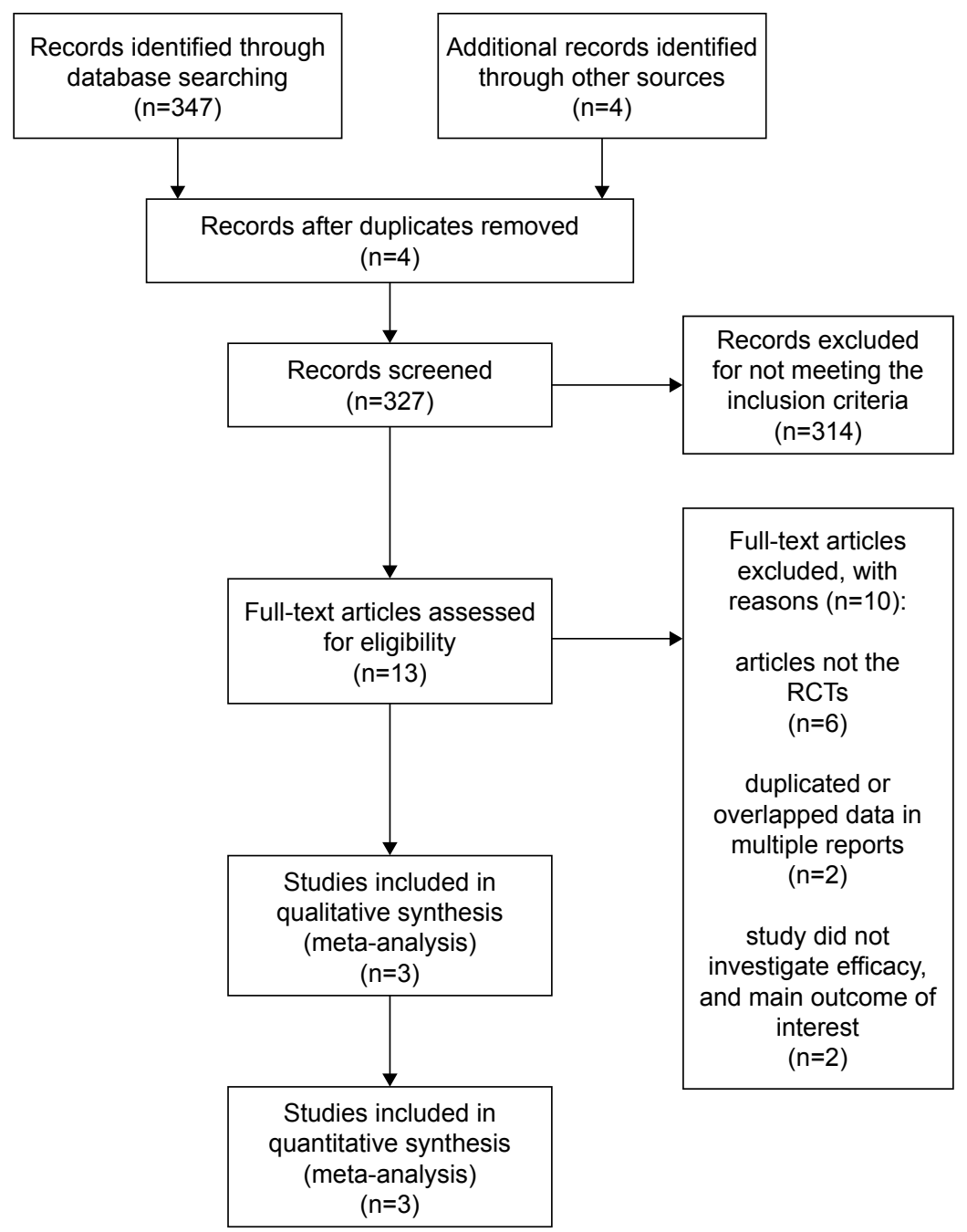

Figure I PRISMA flow chart of the selection process for identification of eligible studies for pooling.

Abbreviations: PRISMA, Preferred Reporting Items for Systematic Reviews and Meta-Analyses; RCT, randomized controlled trial.

The pooled data showed that nivolumab plus chemotherapy did not improve OS (OR: 0.77, 95\% CI: 0.57-1.03, $P=0.08$ ) over chemotherapy (Figure 3).

Pooled analysis of ORR comparing the addition of nivolumab with chemotherapy

Pooling ORR data ${ }^{14-16}$ did not improve efficacy for nivolumab (OR: $1.40,95 \%$ CI: $0.66-2.96, P=0.39$ ). In other words, the addition of nivolumab did not increase the ORR (Figure 4).

Pooled analysis of SAEs comparing the addition of nivolumab with chemotherapy

SAE data were available for the three RCTs. ${ }^{14-16}$ Results showed much worse (grade 3-5 adverse events) SAEs in the nivolumab group than in the chemotherapy group (OR: 0.13 ,

Table I The primary characteristics of the eligible studies in more detail

\begin{tabular}{|c|c|c|c|c|c|c|c|c|}
\hline Study & Year & Trial name & $\begin{array}{l}\text { Trial } \\
\text { phase }\end{array}$ & Stage & Histology & $\begin{array}{l}\text { PD-LI tumor } \\
\text { expression level }\end{array}$ & Study arm (N) & $\begin{array}{l}\text { Comparative } \\
\operatorname{arm}(\mathbf{N})\end{array}$ \\
\hline $\begin{array}{l}\text { Brahmer } \\
\text { et al }{ }^{15}\end{array}$ & 2015 & CheckMate 017 & 3 & IIlb/IV & Squamous & $\geq 1 \%, \geq 5 \%$, and $\geq 10 \%$ & $\begin{array}{l}\text { Nivolumab } 3 \text { mg/kg } \\
\text { every } 2 \text { weeks }(n=135)\end{array}$ & $\begin{array}{l}\text { Docetaxel } 75 \mathrm{mg} / \mathrm{m}^{2} \\
\text { every } 3 \text { weeks }(\mathrm{n}=137)\end{array}$ \\
\hline $\begin{array}{l}\text { Borghaei } \\
\text { et } \mathrm{al}^{14}\end{array}$ & 2015 & CheckMate 057 & 3 & IIIb/IV & Nonsquamous & $\geq 1 \%, \geq 5 \%$, and $\geq 10 \%$ & $\begin{array}{l}\text { Nivolumab } 3 \text { mg/kg } \\
\text { every } 2 \text { weeks }(n=292)\end{array}$ & $\begin{array}{l}\text { Docetaxel } 75 \mathrm{mg} / \mathrm{m}^{2} \\
\text { every } 3 \text { weeks }(\mathrm{n}=290)\end{array}$ \\
\hline $\begin{array}{l}\text { Carbone } \\
\text { et } \mathrm{al}^{16}\end{array}$ & 2017 & CheckMate 026 & 3 & IV or recurrent & $\begin{array}{l}\text { Squamous and } \\
\text { nonsquamous }\end{array}$ & $\geq 1 \%$ and $\geq 5 \%$ & $\begin{array}{l}\text { Nivolumab } 3 \text { mg/kg } \\
\text { every } 2 \text { weeks }(n=27 \mathrm{I})\end{array}$ & $\begin{array}{l}\text { Investigator's choice of } \\
\text { platinum-based doublet } \\
\text { chemotherapy }(n=270)\end{array}$ \\
\hline
\end{tabular}




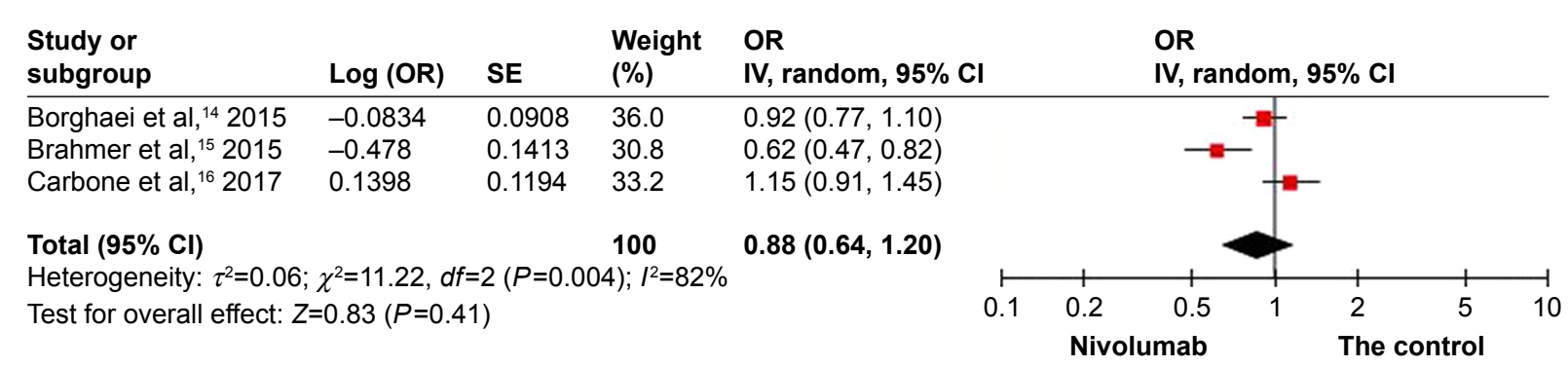

Figure 2 Pooled analysis of PFS comparing the addition of nivolumab with chemotherapy. Abbreviations: OR, odds ratio; PFS, progression-free survival.

95\% CI: $0.09-0.17, P<0.00001$ ) (Figure 5). Subgroup meta-analysis of PFS and OS in patients with tumor PD-L1 expression levels $\geq 5 \%$ demonstrated that nivolumab therapy did not prolong PFS (OR: 0.84, 95\% CI: 0.70-1.00, $P=0.05$ ) (Figure 6) or OS (OR: 0.63, 95\% CI: 0.34-1.15, $P=0.13$ ) (Figure 7).

\section{Discussion}

NSCLC is the main cause of cancer-related mortality worldwide. ${ }^{5}$ Chemotherapy has provided modest improvements in the survival of patients with advanced NSCLC. Compared to traditional antineoplastic therapies, immune checkpoint receptors are able to target tumor cells and have been reported to be important in future cancer treatment. ${ }^{19}$ PD- 1 is a vital immune checkpoint receptor, which is expressed on activated T-cells ${ }^{20}$ and has been demonstrated to be a valuable clinical target for cancer treatment. Based on results with nivolumab, anti-PD-1/PD-L1 therapy is a highly promising treatment for patients with advanced NSCLC. However, inconsistent results with nivolumab cannot be ignored. ${ }^{14-16}$ Thus, this meta-analysis evaluated the efficacy and safety of nivolumab in advanced NSCLC patients.

In this analysis, the superiority of nivolumab therapy compared to chemotherapy for NSCLC patients was not found for OS, ORR, or PFS, even when limited to the
PD-L1 $\geq 5 \%$ subpopulation. These results must be considered with caution. First, previous studies of nivolumab therapy for NSCLC included squamous and nonsquamous cell subtypes, and it has been shown that anti-PD-1/PD-L1 therapy may provide differential benefit for different histological subtypes. Data from the Cancer Genome Atlas Research Network showed that significant differences may be caused by the mutational complexity between adenocarcinoma and squamous cell lung cancers. ${ }^{21,22}$

The majority of lung adenocarcinomas have undergone complex oncogene mutations, such as in the EGFR gene, ROS1 gene, and TKIs. ${ }^{22}$ However, no similar driver mutations have been identified for squamous cell lung cancers. ${ }^{21}$ As such, platinum-based doublet treatment has been accepted as first-line treatment for squamous NSCLC. ${ }^{23}$ Second, for an individual patient, gene data-based analysis would provide a more precise estimate of the effect of nivolumab. The findings of this study may be due to a high rate of NSCLC harboring a K-ras-mutation. Subgroup analysis for the CheckMate-057 Trial ${ }^{15}$ showed that patients with K-ras-mutations treated with nivolumab had a significant benefit in OS. Mechanistically, NSCLCs harboring K-ras mutations are associated with a higher mutational load and as such are more immunogenic. An increased mutational load may provide for increased efficacy of nivolumab in combination with carboplatin and

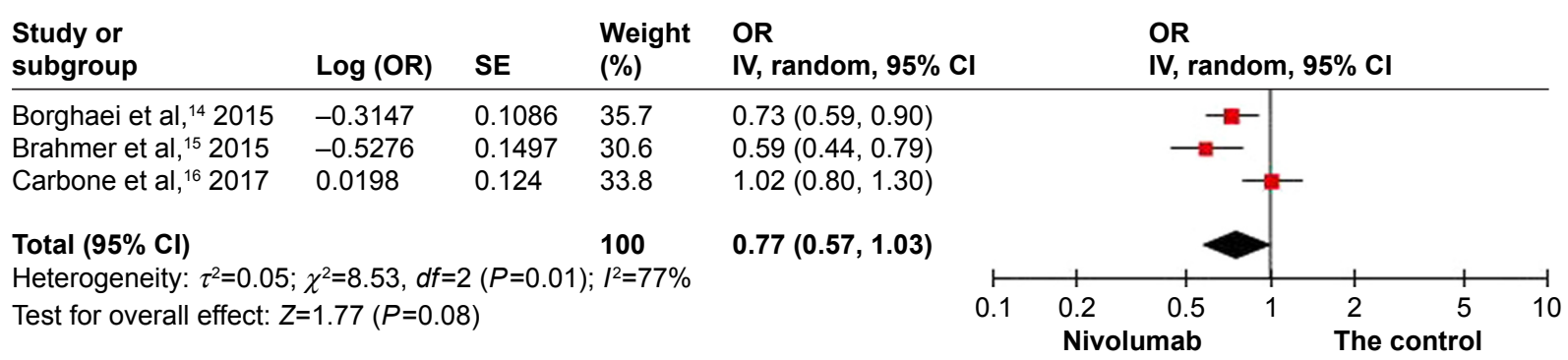

Figure 3 Pooled analysis of OS comparing the addition of nivolumab with chemotherapy. Abbreviations: OR, odds ratio; OS, overall survival. 


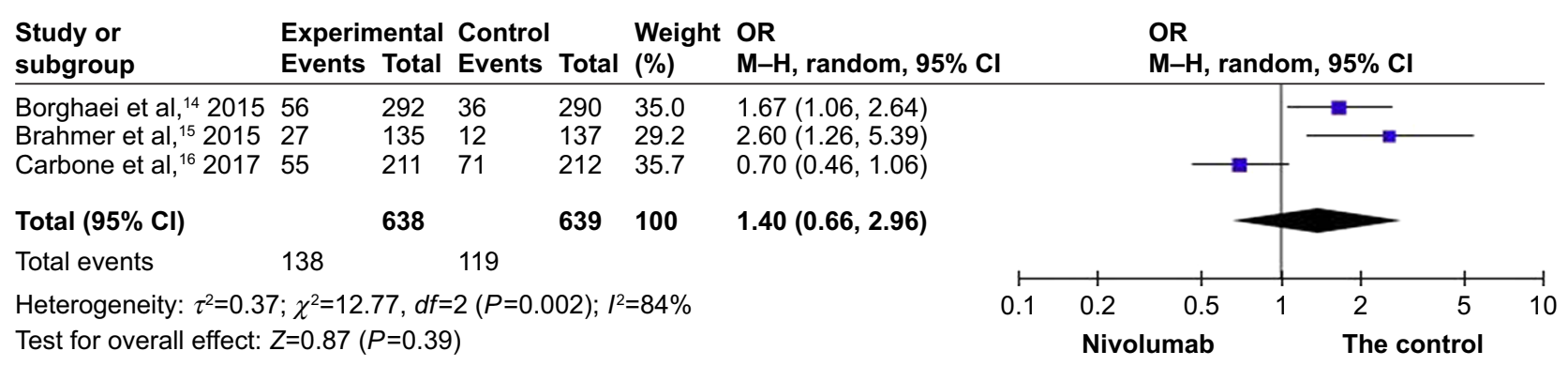

Figure 4 Pooled analysis of ORR comparing the addition of nivolumab with chemotherapy.

Abbreviations: OR, odds ratio; ORR, overall response rate.

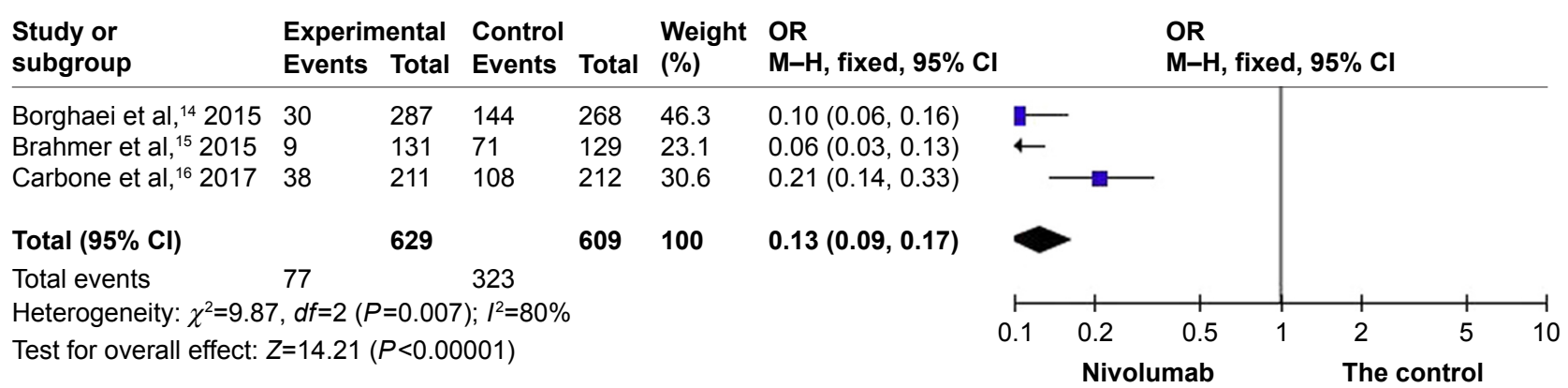

Figure 5 Pooled analysis of SAEs comparing the addition of nivolumab with chemotherapy.

Abbreviations: OR, odds ratio; SAE, serious adverse effect.

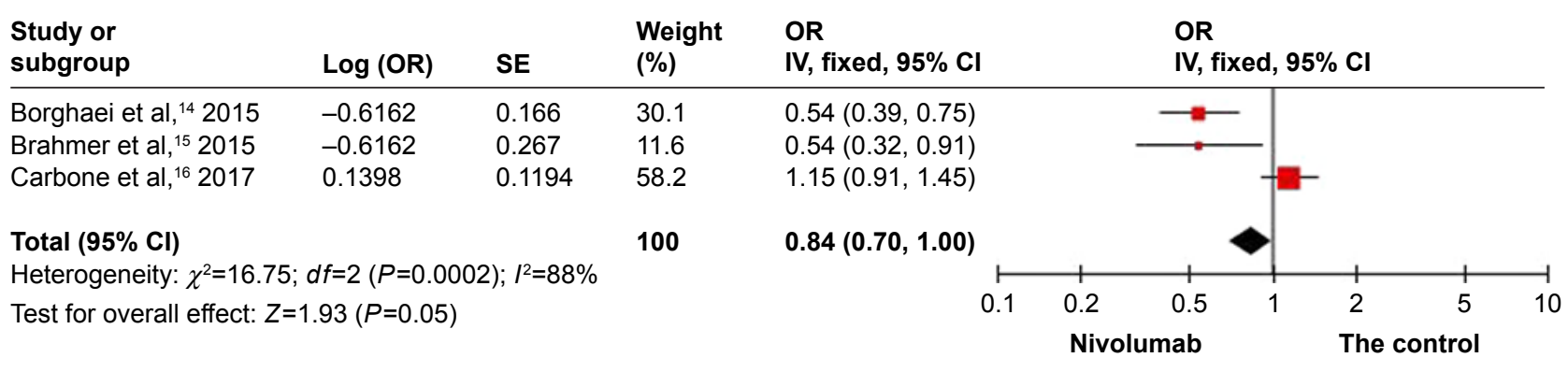

Figure 6 Subgroup meta-analysis of PFS among patients with PD-LI expression level of $\geq 5 \%$. Abbreviations: OR, odds ratio; PFS, progression-free survival.

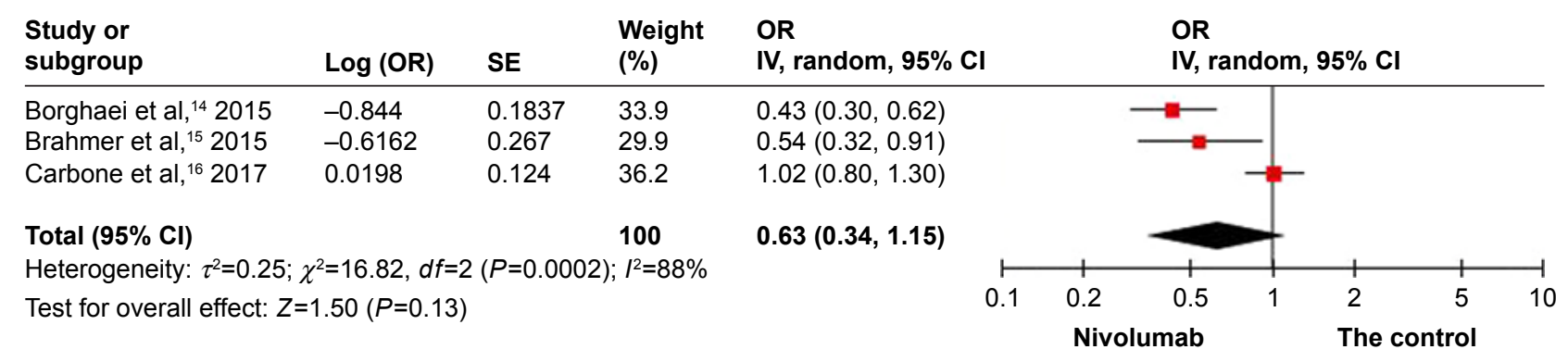

Figure 7 Subgroup meta-analysis of OS among patients with PD-LI expression level of $\geq 5 \%$.

Abbreviations: OR, odds ratio; OS, overall survival. 
paclitaxel due to the higher expression of PD-L1. Third, due to the heterogeneous nature of the included studies, bias may exist, and this may impact outcomes comparison. Pooled results are not optimal and may be questioned.

Early-stage trials have suggested that expression of PD-L1 on tumor cells or tumor-infiltrating lymphocytes (or both) may be worthwhile targets of the PD-1 pathway, serving as potential biomarkers, ${ }^{24,25}$ although the best cutoff scores have not been defined. Further trials are needed to determine the level of PD-L1 expression that provides maximal patient efficacy with a manageable nivolumab safety profile. However, in this analysis there was no difference among patients with PD-L1 expression level of $\geq 5 \%$ in OS or PFS.

The CheckMate-017 trial $^{14}$ demonstrated PD-L1 therapy to have no benefit for squamous NSCLC at any PD-L1 level. The CheckMate-057 trial ${ }^{15}$ showed no significant benefit for nivolumab with regard to ORR, PFS, or OS for nonsquamous cell patients at different cut off scores, although there was a clear escalating clinical benefit with increasing PD-L1 expression levels. ${ }^{26}$

The tumor microenvironment is complicated. Colocalization of PD-L1 and tumor-infiltrating lymphocytes have been reported to be predictive of a protective antitumor response. ${ }^{27}$ PD-L1 expression by other tumor-infiltrating immune cells can also affect a protective response. ${ }^{28}$ Additional studies will be necessary to establish a relationship between PD-L1 expression and a patient's response to nivolumab treatment.

Immune-treatment adverse events are generally infrequent and less severe than other forms of cancer treatment. In this study, nivolumab showed comparatively few adverse events but did include pneumonitis, which was infrequent, of low severity, and acceptable by established guidelines. Overall, adverse events due to immunotherapies, such as nivolumab, differ from those seen with traditional cytotoxic drugs. Hence, such immunotherapies should be quickly advanced for rapid evaluation and the initiation of treatment.

\section{Conclusion}

In conclusion, nivolumab monotherapy for patients with advanced NSCLC was generally well tolerated, with promising antitumor activity and a manageable safety profile.

More RCTs with larger sample sizes are needed to detect relevant biomarkers that have sufficient sensitivity and specificity to predict patient populations that would most benefit from nivolumab, in particular those patients with pretreated and advanced NSCLC.

\section{Disclosure}

The authors report no conflicts of interest in this work.

\section{References}

1. Travis WD. Pathology of lung cancer. Clin Chest Med. 2011;32(4): 669-692.

2. Spiro SG, Rudd RM, Souhami RL, et al. Chemotherapy versus supportive care in advanced non-small-cell lung cancer: improved survival without detriment to quality of life. Thorax. 2004;59(10):828-836.

3. NSCLC Meta-Analyses Collaborative Group. Chemotherapy in addition to supportive care improves survival in advanced non-small-cell lung cancer: a systematic review and meta-analysis of individual patient data from 16 randomized controlled trials. J Clin Oncol. 2008;26(28):4617-4625.

4. Chang S, Dai M, Ren JS, Chen YH, Guo LW. Estimates and prediction on incidence, mortality and prevalence of lung cancer in China in 2008. Zhonghua Liu Xing Bing Xue Za Zhi. 2012;33(4):391-394.

5. Wood DE. National Comprehensive Cancer Network (NCCN) Clinical Practice Guidelines for Lung Cancer Screening. Thorac Surg Clin. 2015;25(2):185-197.

6. Sandler A, Gray R, Perry MC, et al. Paclitaxel-carboplatin alone or with bevacizumab for non-small-cell lung cancer. $N$ Engl $J$ Med. 2006;355(24):2542-2550.

7. Reck M, Kaiser R, Mellemgaard A, et al. Docetaxel plus nintedanib versus docetaxel plus placebo in patients with previously treated non-small-cell lung cancer (LUME-Lung 1): a phase 3, double-blind, randomised controlled trial. Lancet Oncol. 2014;15(2):143-155.

8. Gettinger SN, Horn L, Gandhi L, et al. Overall Survival and Long-Term Safety of Nivolumab (Anti-Programmed Death 1 Antibody, BMS936558, ONO-4538) in Patients With Previously Treated Advanced NonSmall-Cell Lung Cancer. J Clin Oncol. 2015;33(18):2004-2012.

9. Larkin J, Lao CD, Urba WJ, et al. Efficacy and Safety of Nivolumab in Patients With BRAF V600 Mutant and BRAF Wild-Type Advanced Melanoma: A Pooled Analysis of 4 Clinical Trials. JAMA Oncol. 2015; 1(4):433-440.

10. Pardoll DM. The blockade of immune checkpoints in cancer immunotherapy. Nat Rev Cancer. 2012;12(4):252-264.

11. Schadendorf D, Hodi FS, Robert C, et al. Pooled Analysis of LongTerm Survival Data From Phase II and Phase III Trials of Ipilimumab in Unresectable or Metastatic Melanoma. J Clin Oncol. 2015;33(17): 1889-1894.

12. Topalian SL, Sznol M, Mcdermott DF, et al. Survival, durable tumor remission, and long-term safety in patients with advanced melanoma receiving nivolumab. J Clin Oncol. 2014;32(10):1020-1030.

13. Pirker R, Pereira JR, Szczesna A, et al. Cetuximab plus chemotherapy in patients with advanced non-small-cell lung cancer (FLEX): an open-label randomised phase III trial. Lancet. 2009;373(9674): $1525-1531$

14. Borghaei H, Paz-Ares L, Horn L, et al. Nivolumab versus Docetaxel in Advanced Nonsquamous Non-Small-Cell Lung Cancer. N Engl J Med. 2015;373(17):1627-1639.

15. Brahmer J, Reckamp KL, Baas P, et al. Nivolumab versus Docetaxel in Advanced Squamous-Cell Non-Small-Cell Lung Cancer. $N$ Engl $J$ Med. 2015;373(2):123-135.

16. Carbone DP, Reck M, Paz-Ares L, et al. First-Line Nivolumab in Stage IV or Recurrent Non-Small-Cell Lung Cancer. $N$ Engl J Med. 2017;376(25):2415-2426.

17. Higgins JP, Thompson SG. Quantifying heterogeneity in a metaanalysis. Stat Med. 2002;21(11):1539-1558.

18. Higgins JP, Thompson SG, Deeks JJ, Altman DG. Measuring inconsistency in meta-analyses. BMJ. 2003;327(7414):557-560.

19. Domingues D, Turner A, Silva MD, et al. Immunotherapy and lung cancer: current developments and novel targeted therapies. Immunotherapy. 2014;6(11):1221-1235. 
20. Katsuya Y, Horinouchi H, Asao T, et al. Expression of programmed death 1 (PD-1) and its ligand (PD-L1) in thymic epithelial tumors: Impact on treatment efficacy and alteration in expression after chemotherapy. Lung Cancer. 2016;99:4-10.

21. Cancer Genome Atlas Research Network. Comprehensive genomic characterization of squamous cell lung cancers. Nature. 2012;489(7417): 519-525

22. Cancer Genome Atlas Research Network. Comprehensive molecular profiling of lung adenocarcinoma. Nature. 2014;511(7511):543-550.

23. Ang YL, Tan HL, Soo RA. Best practice in the treatment of advanced squamous cell lung cancer. Ther Adv Respir Dis. 2015;9(5):224-235.

24. Topalian SL, Hodi FS, Brahmer JR, et al. Safety, activity, and immune correlates of anti-PD-1 antibody in cancer. NEngl J Med. 2012;366(26): 2443-2454.

25. Taube JM, Klein A, Brahmer JR, et al. Association of PD-1, PD-1 ligands, and other features of the tumor immune microenvironment with response to anti-PD-1 therapy. Clin Cancer Res. 2014;20(19): 5064-5074.
26. Horn L, Spigel DR, Vokes EE, et al. Nivolumab Versus Docetaxel in Previously Treated Patients With Advanced Non-Small-Cell Lung Cancer: Two-Year Outcomes From Two Randomized, Open-Label, Phase III Trials (CheckMate 017 and CheckMate 057). J Clin Oncol. 2017;35(35):3924-3933.

27. Taube JM, Anders RA, Young GD, et al. Colocalization of inflammatory response with B7-h1 expression in human melanocytic lesions supports an adaptive resistance mechanism of immune escape. Sci Transl Med. 2012;4127(127):ra137.

28. Herbst RS, Soria JC, Kowanetz M, et al. Predictive correlates of response to the anti-PD-L1 antibody MPDL3280A in cancer patients. Nature. 2014;515(7528):563-567.

\section{Publish your work in this journal}

OncoTargets and Therapy is an international, peer-reviewed, open access journal focusing on the pathological basis of all cancers, potential targets for therapy and treatment protocols employed to improve the management of cancer patients. The journal also focuses on the impact of management programs and new therapeutic agents and protocols on

\section{Dovepress}

patient perspectives such as quality of life, adherence and satisfaction. The manuscript management system is completely online and includes a very quick and fair peer-review system, which is all easy to use. Visit http://www.dovepress.com/testimonials.php to read real quotes from published authors.

Submit your manuscript here: http://www.dovepress.com/oncotargets-and-therapy-journal 